\title{
THE SCHNEIDER-LANG THEOREM FOR FUNCTIONS WITH ESSENTIAL SINGULARITIES
}

\author{
JACK DIAMOND
}

\begin{abstract}
A new proof of Schwarz's lemma for functions with a finite number of essential singularities is given. The proof is valid for $p$-adic as well as complex functions and is used to extend Bertrand's version of the Schneider-Lang theorem for $p$-adic functions with one, common, finite singularity to functions with finitely many singularities.
\end{abstract}

1. Introduction. The Schneider-Lang theorem gives a maximum for the number of points at which a family of meromorphic functions satisfying certain conditions can simultaneously take on values in a number field. D. Bertrand has extended it to include the case of a family of complex functions having a finite set of essential singularities and to the case of a family of $p$-adic functions having a common essential singularity. These generalizations are based on an extension of Schwarz's lemma to functions with singularities. Schwarz's lemma is a refinement of the maximum modulus principle in the case where a function is known to have many zeroes.

We have another, more algebraic, approach to Schwarz's lemma that only uses analytic concepts which apply to both complex and $p$-adic functions. Consequently, we will give a unified proof of the Schneider-Lang theorem which is more elementary than Bertrand's in the complex case and extends his result in the p-adic case.

$\mathbf{C}$ is the field of complex numbers and $\mathbf{C}_{p}$ is the completion of the algebraic closure of the $p$-adic field $\mathbf{Q}_{p}$. $\mathbf{C}^{\prime}$ will denote both $\mathbf{C} \cup\{\infty\}$ and $\mathbf{C}_{p} \cup\{\infty\} .\left|\mathbf{C}^{\prime}\right|$ is the set of values of $|x|$ for $x \in \mathbf{C}^{\prime}, x \neq \infty$. If $\mathbb{Q}=\left\{a_{1}, \ldots, a_{k}\right\}$ is a finite subset of $C^{\prime}$, then as a concept of analyticity on $C^{\prime}-\mathbb{Q}$ we use the property that $f(x)=$ $\Sigma_{1<i<k} f_{i}(x)$ where $f_{i}(x)$ is a power series in $x$ if $a_{i}=\infty$ and a power series in $\left(x-a_{i}\right)^{-1}$ if $a_{i} \neq \infty$. In the $p$-adic case this is not as general as Krasner's concept of analyticity. The restriction is made in order to use the maximum modulus principle on disc-shaped domains, a principle, which, as is shown in [4], does not apply to (Krasner) analytic functions.

$C(a, r)$ is the circle with center $a$ and radius $r$ if $a \neq \infty$ and the circle with center 0 and radius $1 / r$ if $a=\infty$. If $a=\infty$ the usual exterior of $C(0,1 / r)$ will be called the interior. Given a family of circles $C\left(a_{i}, r_{i}\right)$ in which each $r_{i}$ is a function of $r$, we let $\mathscr{D}(r)=\cup_{i} C\left(a_{i}, r_{i}\right)$ and $m_{f}(r)=\sup _{x \in \mathscr{D}(r)}|f(x)|$.

Received by the editors November 26, 1979; presented to the Society, August 24, 1979.

AMS (MOS) subject classifications (1970). Primary 10F35, $12 \mathrm{~B} 40$.

Key words and phrases. Schwarz's lemma, Schneider-Lang theorem. 
2. Schwarz's lemma. This version of Schwarz's lemma is similar to Bertrand's complex version in [1] and extends his $p$-adic version in [2] to functions with finitely many singularities.

TheOREM (SCHWARZ'S Lemma). Suppose that $f$ is analytic on $\mathbf{C}^{\prime}-\mathbb{Q}, \mathbb{Q}=$ $\left\{a_{1}, \ldots, a_{k}\right\}, f \not \equiv 0, f$ has zeroes at $z_{1}, \ldots, z_{h}$ (not necessarily distinct), $\mathcal{Z}$ is the set of distinct elements among $z_{1}, \ldots, z_{h}, \mathscr{W}=\left\{w_{1}, \ldots, w_{k}\right\}$ is a set of positive rational numbers whose sum is $W, R_{i}=R^{1 / w_{i}}$ and $r$ is chosen small enough that the circles $C\left(a_{i}, r_{i}\right)$ do not have any points of $\mathscr{Z}$ or of $\mathscr{Q}$, excepting the given center, on or within themselves. Then, if $R$ and $r \in\left|\mathbf{C}^{\prime}\right|$ and $0<R<r$, there is a constant $c$ depending only on $\mathcal{Q}, \mathcal{W}, \mathscr{Z}$ and $r$ so that

$$
m_{f}(r)<c^{h}(R / r)^{h / w^{\prime}} m_{f}(R) \text {. }
$$

PRoof. First, suppose that $W$ is a set of positive integers. Then, $f^{W}(x)=$ $g(x) \varphi(x)$ where

$$
\varphi(x)=\frac{\Pi_{1<j<h}^{*}\left(x-z_{j}\right)^{W}}{\Pi_{1<i<k}^{*}\left(x-a_{i}\right)^{h w_{i}}} .
$$

The * in the expression for $\varphi$ indicates that if $a_{i}$ or $z_{j}$ is $\infty$ the corresponding factor should be omitted.

The point of this factorization for $f^{W}(x)$ is that $g$ is also analytic on $\mathbf{C}^{\prime}-\mathscr{Q}$ and $|\varphi(x)|$ can be easily estimated.

We will find upper and lower bounds for $|\varphi(x)|$ and then apply the maximum modulus principle to $g$ to obtain Schwarz's lemma. The maximum modulus principle in this situation is that $m_{g}(r) \leqslant m_{g}(R)$. In the $p$-adic case this is a consequence of the maximum modulus principle on discs.

In order to estimate $|\varphi(x)|, x \in \mathscr{D}(R)$, it is necessary to consider $a_{i}=\infty$ separately from the other $a_{i} . r$ will be fixed throughout and $R<r$.

Case 1. $a_{I} \neq \infty, x \in C\left(a_{I}, R_{I}\right)$,

$$
|\varphi(x)|=\frac{R^{-h} \Pi_{1<j<h}^{*}\left|x-z_{j}\right|^{W}}{\Pi_{1<i<k, i \neq l}^{*}\left|x-a_{i}\right|^{h w_{i}}} .
$$

Clearly, $\left|x-z_{j}\right|$ and $\left|x-a_{j}\right|$ are bounded above by a number depending only on $\mathcal{Q}, \mathcal{Z}$, $W$ and $r$.

On the other hand, $\left|x-z_{j}\right|$ and $\left|x-a_{i}\right|$ are bounded below by $m-r_{I}$, where $m$ is the minimum distance between two finite points in $\mathscr{Q} \cup \mathscr{Z}$.

The next step depends on whether or not $\infty \in \mathbb{Q}$. The calculations are similar, though the possibility of $z_{j}=\infty$ needs to be taken into account if $\infty$ is not a singular point. Suppose $a_{J}=\infty$ and $M$ is the maximum distance between two finite points in $\mathbb{Q} \cup \mathcal{Z}$. Then

$$
\frac{R^{-h}\left(m-r_{I}\right)^{h W}}{\left(M+r_{I}\right)^{h\left(W-w_{I}-w_{J}\right)}}<|\varphi(x)|<\frac{R^{-h}\left(M+r_{I}\right)^{h W}}{\left(m-r_{I}\right)^{h\left(W-w_{I}-w_{J}\right)}}
$$

and $0<R^{-h} A_{I}^{h}<|\varphi(x)|<R^{-h} B_{I}^{h}$ with

$$
A_{I}=\frac{\left(m-r_{I}\right)^{W}}{\left(M+R_{I}\right)^{W-w_{I}-w_{J}}}, \quad B_{I}=\frac{\left(M+r_{I}\right)^{W}}{\left(m-r_{I}\right)^{W-w_{I}-w_{J}}} .
$$


Case 2. $a_{I}=\infty, x \in C\left(\infty, R_{I}\right)$

$$
|\varphi(x)|=\frac{\Pi_{1<j<h}^{*}\left|x-z_{j}\right|^{W}}{\Pi_{1<i<k}^{*}\left|x-a_{i}\right|^{h w_{i}}} .
$$

Let $M=\max \left\{\left|a_{i}\right|,\left|z_{j}\right| \mid a_{i} \neq \infty\right\}$. Then

$$
1 / r_{I}-M<\left|x-z_{j}\right|<1 / R_{I}+M, \quad 1 / r_{I}-M<\left|x-a_{i}\right|<1 / R_{I}+M
$$

and

$$
\frac{\left(1 / R_{I}-M\right)^{h W}}{\left(1 / R_{I}+M\right)^{h\left(W-w_{I}\right)}}<|\varphi(x)|<\frac{\left(1 / R_{I}+M\right)^{h W}}{\left(1 / R_{I}-M\right)^{h\left(W-w_{I}\right)}} .
$$

Hence, $0<R^{-h} A_{I}^{h}<|\varphi(x)|<R^{-h} B_{I}^{h}$ with

$$
A_{I}=\frac{\left(1-M r_{I}\right)^{W}}{\left(1+M r_{I}\right)^{W-w_{I}}}, \quad B_{I}=\frac{\left(1+M r_{I}\right)^{W}}{\left(1-M r_{I}\right)^{W-w_{I}}}
$$

If $A=\min _{I}\left\{A_{I}\right\}$ and $B=\max _{I}\left\{B_{I}\right\}$, then when $x \in \mathscr{D}(R)$

$$
0<R^{-h} A^{h}<|\varphi(x)|<R^{-h} B^{h}
$$

Since $f^{W}(x)=g(x) \varphi(x)$, it follows that

$$
R^{-h} A^{h}|g(x)| \leqslant\left|f^{W}(x)\right|<R^{-h} B^{h}|g(x)|
$$

and

$$
0<R^{-h} A^{h} m_{g}(R)<m_{f^{w}}(R)<R^{-h} B^{h} m_{g}(R) .
$$

Hence, since $R \leqslant r$,

$$
\begin{aligned}
m_{f}^{w}(r) & <r^{-h} B^{h} m_{g}(r) \leqslant r^{-h} B^{h} m_{g}(R) \\
& =(R / r)^{h}(B / A)^{h} A^{h} R^{-h} m_{g}(R)<(R / r)^{h}(B / A)^{h} m_{f} w(R) .
\end{aligned}
$$

Choosing $c=(B / A)^{1 / W}$ and taking the $W$ th root of each side of the above inequality yields

$$
m_{f}(r)<(R / r)^{h / w^{h}} c^{h} m_{f}(R)
$$

If the $w_{i}$ are rational numbers with a common denominator of $d$, then the $R_{i}=R^{1 / w_{i}}$ have the form $\left(R^{d}\right)^{1 / u_{i}}$ with $u_{i}$ an integer. The theorem now follows from the case for integral $u_{i}$ already proved.

3. The Schneider-Lang theorem. Before stating the Schneider-Lang theorem we will recall the definition for finite order of growth of a function at an isolated singular point. If $a \neq \infty$, then $f$ has order of growth $<w$ at $a$ if there is a neighborhood around $a$ and a constant $A$ so that $|f(x)|<\exp \left(A R^{-w}\right)$ when $|x-a|=R$. If $a=\infty$, then $f$ has order of growth $<w$ at $a$ if there is a neighborhood around $a$ and a constant $A$ so that $|f(x)|<\exp \left(A R^{w}\right)$ when $|x|=R$.

If $\mathbb{Q}=\left\{a_{1}, \ldots, a_{k}\right\}$ is a finite subset of $\mathbf{C}^{\prime}$ and $\mathscr{W}=\left\{w_{1}, \ldots, w_{k}\right\}$ is a set of positive real numbers, let $\mathscr{R}(\mathscr{Q}, \mathscr{W})$ be the field of fractions of the ring of functions which are analytic on $\mathbf{C}^{\prime}-\mathbb{Q}$ and have order $<w_{i}$ at $a_{i}, i=1, \ldots, k$.

The Schneider-Lang theorem is given in [3] for functions meromorphic on $\mathbf{C}$. 
Bertrand generalized the theorem to complex functions with a finite number of essential singularities in [1] and to $p$-adic functions with one common finite singularity in [2]. It will be shown below that Bertrand's result for complex functions also holds for $p$-adic functions.

TheOREM. Suppose that $Q$ is a set of $k$ points of $\mathbf{C}^{\prime}, f_{1}, \ldots, f_{N}$ are mappings from $\mathbf{C}^{\prime}-\mathcal{Q}$ into $\mathbf{C}^{\prime}$, W is a set of $k$ positive rational numbers whose sum is $W, \mathbf{K}$ is a number field embedded in $\mathbf{C}^{\prime}$ and the following conditions are satisfied.

(i) $f_{1}$ and $f_{2}$ are algebraically independently over $\mathbf{K}$.

(ii) $d / d x$ maps $\mathbf{K}\left[f_{1}, \ldots, f_{N}\right]$ into itself.

(iii) There is a set of distinct points $z_{1}, \ldots, z_{m}$ such that $f_{i}\left(z_{j}\right) \in \mathbf{K}$ for $i=$ $1, \ldots, N$ and $j=1, \ldots, m$.

(iv) $f_{i} \in \mathscr{R}(\mathcal{Q}$, QS ) for $i=1, \ldots, N$.

Then $m<2 W[\mathbf{K}: \mathbf{Q}]$.

The proof is a standard argument which appears in several of the references. We will give an outline to show how Schwarz's lemma is applied.

Given a positive integer $S$, a function $F_{S}$, not identically zero, is constructed as a polynomial in $f_{1}$ and $f_{2} . F_{S}$ has the property that it has a zero of order at least $S$ at each $z_{j}, j=1, \ldots, m$. Suppose that for each $j D^{n} F_{S}\left(z_{j}\right)=0$ if $n<\sigma$ and for some $J D^{\sigma} F_{S}\left(z_{J}\right) \neq 0 . \sigma \geqslant S$. Let $\gamma_{\sigma}=D^{\sigma} F_{S}\left(z_{J}\right) / \sigma$ !.

On the basis of hypotheses (i), (ii) and (iii) a lower bound for $\log \left|\gamma_{\sigma}\right|$ can be obtained,

$$
\log \left|\gamma_{\sigma}\right| \geqslant-([\mathbf{K}: \mathbf{Q}]+o(1)) \sigma \log \sigma
$$

where $o(1)$ approaches 0 as $\sigma$ approaches $\infty$.

In order to obtain an upper bound for $\log \left|\gamma_{\sigma}\right|$, Cauchy's theorem is used to change the problem to that of bounding $\left|F_{S}(x)\right|$ on a small circle around $z_{J}$. Now, Schwarz's lemma with $r$ fixed, $R=\sigma^{-1 / 2}, R_{i}=R^{1 / w_{i}}$ and $h=m \sigma$ is used together with hypothesis (iv) to show that

$$
\log \left|\gamma_{\sigma}\right|<(-m / 2 W) \sigma \log \sigma+o(\sigma \log \sigma) .
$$

When the upper and lower bounds for $\log \left|\gamma_{o}\right|$ are compared we find $m<$ $2 W[\mathbf{K}: \mathbf{Q}]$.

\section{REFERENCES}

1. D. Bertrand, Un théorème de Schneider-Lang sur certains domaines non simplement connexes, Séminaire Delange-Pisot-Poitou (16 année: 1974/75), Théorie des Nombres, Fasc. 2, Exp. No. G-18, Secrétariat Mathématique, Paris, 1975.

2. __ Séries d'Eisenstein et transcendance, Bull. Soc. Math. France, 104 (1976), 309-321.

3. S. Lang, Introduction to transcendental numbers, Addison-Wesley, Reading, Mass., 1966.

4. P. Robba and A. Escassut, Prolongement analytique et algèbres de Banach ultrametrique, Astérisque 10, Soc. Math. France, 1973.

5. M. Waldschmidt, Nombres transcendants, Lecture Notes in Math., vol. 402, Springer-Verlag, Berlin, 1974.

Department of Mathematics, Queens College, Flushing, New York 11367 